\title{
PHOTOGRAPHIC WIDE-FIELD IMAGING FROM SCHMIDT PLATES - II
}

\author{
H. ZODET, J. QUEBATTE and H.-H. HEYER \\ European Southern Observatory \\ Garching \\ Germany
}

\section{Combination of 'Unsharp Masking' and 'Image Amplification'}

For this work, we used the red plates of POSS II fields 340 and 115 which show the impressive wide-field areas around the IC 1795/1805 complex (Plate 1) and Gamma Cygni (Plate 2). The applied photographic method aims at the simultaneous enhancing of very faint features and compression of the dynamic range, so that all features become visible on the same print.

Step 1 (the unsharp mask): Copy the original plate in Contact Printer Agfa-Gevaert SV -400 with attached Durst Digitim 2000 timer on Agfa Graphic Avitone Duplicating Film P3p and process manually in Kodak D-76 $(1+3)$ developer at $21^{\circ} \mathrm{C}$ for 9 minutes.

Step 2 (image amplification of masked original): Copy onto Agfa Rapidoline RA 711-p film and process automatically in an Ifford $2150 \mathrm{RC}$ Automatic B/W Processor.

The reproduced dynamic range is from the sky background (original density $1.2 \mathrm{D}$ ) to well above 3.0 D, i.e. a factor of about 100 in density. The two fields cover approximately 6.1 (Plate 1) and 15.2 square degrees, respectively; with a resolution of 1 arcsec, this corresponds to approximately $8 \cdot 10^{7}$ and $2 \cdot 10^{8}$ pixels. The reproduction procedure takes about 1 day/print.

\section{Scientific Content of the Images}

The first example shows the giant H II regions IC 1795 (= W3; upper left corner) and IC 1805 (=W4; centre) in the red, i.e. many of the structures are mostly seen in the light of Ho. The intricate structure of IC 1805, including filamentary dark nebulae in the fore-front and numerous stellar aggregations, bear witness to the intense star formation in this region. The entire outer shell around IC 1805 has never been shown with this amount of detail. Note also the very faint, large structures in the background outside the nebulae, only visible because of the amplification.

The area around Gamma Cygni, near the galactic plane, demonstrates the usefulness of being able to perceive simultaneously the exceedingly complex structure of the interstellar medium, in and outside bright nebulae. A careful inspection reveals that the 'foreground' structure is very similar all over the field and is therefore likely to be at least partly due to relatively nearby material. 


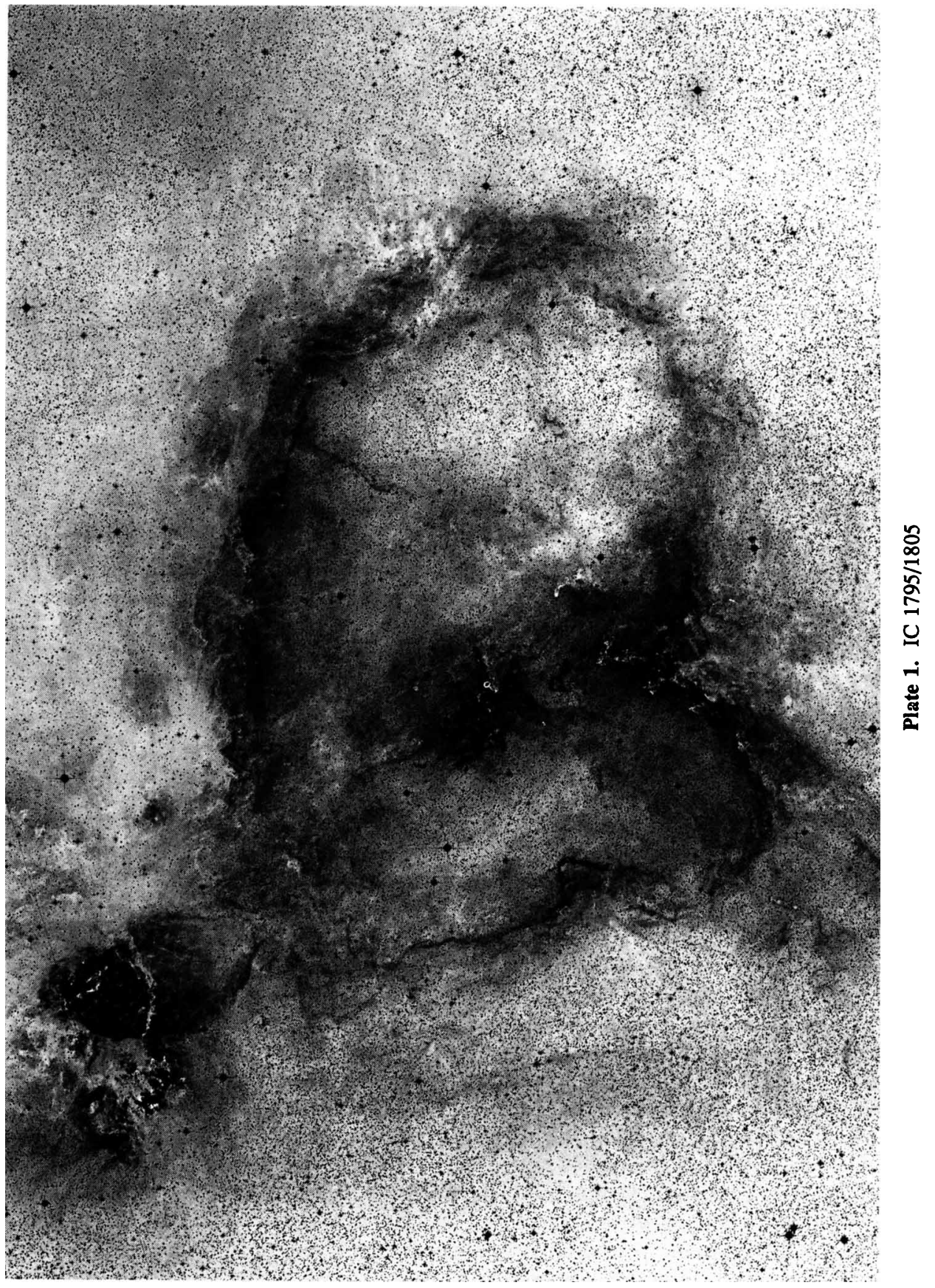




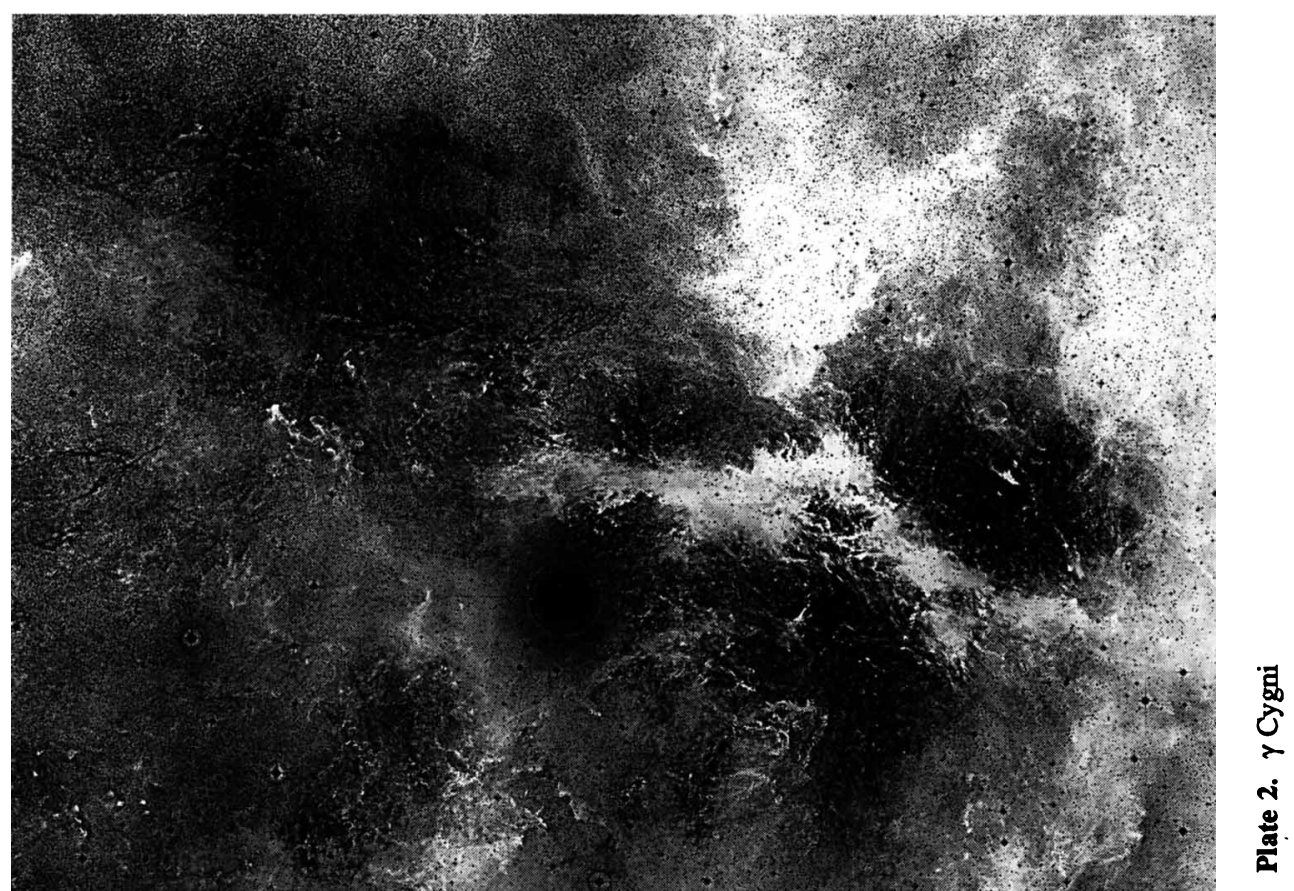

